\title{
Concept Development and Implementation of Family Care/Caring Theory in Concentric Sphere Family Environment Theory
}

\author{
Naohiro Hohashi*, Junko Honda \\ Division of Family Health Care Nursing, Department of Nursing, Graduate School of Health Sciences, \\ Kobe University, Kobe, Japan \\ Email: "naohiro@hohashi.org
}

Received 9 August 2015; accepted 4 September 2015; published 7 September 2015

Copyright (C) 2015 by authors and Scientific Research Publishing Inc.

This work is licensed under the Creative Commons Attribution International License (CC BY). http://creativecommons.org/licenses/by/4.0/

(c) (7) Open Access

\section{Abstract}

Caring is directed toward a variety of things. One of them is thought to be the concept of "family caring" aimed at families. This study attempts to clarify family caring and develop Family Care/ Caring Theory (FCCT), with the aim of implementing it in conjunction with an existing family nursing theory, the Concentric Sphere Family Environment Theory (CSFET). In Japan and in Hong Kong, family ethnography (including formal interviews) was conducted. As a result, the item "family health care nurses and their colleagues" was added to the family external environment of the CSFET. In the family environment, evidence was obtained to the effect that the family system unit is cared for by the nursing professional, and conversely the family system unit cares for the nursing professional, in a circular transaction. Observing the two-dimensional plane formed by the structural distance and functional distance, family caring assumes a structure of concentric circles, and according to transactions, the structural distance and functional distance between the nursing professional and family system unit are gradually approached, and through deepening of mutual trust maintain an appropriate distance. Moreover observing the three-dimensional space-time continuum which is created through addition of the temporal distance, family caring forms a helical structure. As transactions are repeated along the temporal axis, the family system unit's selfactualization of other individuals and the self-actualization of the nursing professional are realized. Through these processes, a family care/caring relationship is reinforced and established. This is the concept of FCCT. Through future utilization in clinical settings this will be empirically substantiated, and it will be necessary to continue making creative corrections and revisions.

\section{Keywords}

Family Caring, Family Care/Caring Theory (FCCT), Concentric Sphere Family Environment Theory

\footnotetext{
${ }^{*}$ Corresponding author.
}

How to cite this paper: Hohashi, N. and Honda, J. (2015) Concept Development and Implementation of Family Care/Caring Theory in Concentric Sphere Family Environment Theory. Open Journal of Nursing, 5, 749-757. 


\section{(CSFET), Family Health Care Nurses and Their Colleagues}

\section{Introduction}

The objects of caring are not only human beings, but can be utilized in transdisciplinary domains so as to include animals, plants, things and ideas [1]. The objects of nursing, in addition to humans, also include families, groups, organizations, communities and so on [2]. In other words, caring that is directed at the family can also be considered within the concept of "family caring." While considerable research has been conducted regarding caring in the field of nursing, most of this involves from one individual to another, in other words, studies that are focused on caring from the nursing professional toward the patient. Consequently, caring assumes a one-way vector and interaction does not take place on a mutual basis [3]. Ray conducted research into caring in the organization of hospitals, from which the unique Theory of Bureaucratic Caring was developed [4]. In this theory, relational caring transcends the bounds of person to person, and occurs in professionals, hospitals and complex health care organizations that are affected by government policies or regulations. In the same manner, this includes hospitals, which are related to major centers of culture, such as cities or nations. However a caring theory does not exist that explains, by treating the family as a single system unit, the mutual caring between the family and nursing professional.

The family [5] that is the target of family nursing is defined as "a unit/organization as a system of the OR operation of individuals, i.e., living people, having the cognition of belonging by other constituent member(s).” The family is a system unit, and family nursing, to realize the well-being of the family system unit, has been established as a specialized field of nursing studies [6]. In the frameworks of family assessment/intervention there are a number of family nursing theories, but none of them devote attention to family caring or focus on transactions between families and nursing professionals. In this study, as one family nursing theory, we propose to conduct concept development of family care/caring that focuses on transactions between the family and nursing professional.

The Concentric Sphere Family Environment Theory (CSFET) proposed by Hohashi [7] is a middle-range family nursing theory that focuses on the family environment that acts on the well-being of the family system unit. The family environment consists of "all entities (people, objects and matters) and phenomena that exist both within and outside the family system unit.” The CSFET takes the form of a three-dimensional logical space-time continuum, consisting of three assessment axes (structural distance, functional distance, and temporal distance). Within these, the positioning of five systems (supra system, macro system, micro system, family internal environment system, and chrono system) enables the family system unit to be viewed in its entirety as a three-dimensional figure.

The supra system is "the external framework that encompasses the entire family environment, which produces the family environment and is directly or indirectly related to the family environment.” This system includes religion, national and regional characteristics, language and so on. The macro system represents "family members' sphere of daily activities, which is situated relatively externally from the family system unit in terms of overall assessment of physical/objective and psychological/subjective aspects.” It includes leisure environment, social resources, workplace environment, politics, economics and so on. The micro system represents "community in the nearby region, which is situated closer to the family system unit in terms of overall assessment of physical/objective and psychological/subjective aspects.” It includes the local living sphere, neighbors, relatives and so on. The above three systems taken together are called the family external environment system. On the other hand, the family internal environment system refers to "the range within the family unit where mutual interactions between family members occur."

Changes in the family external environment system create changes in the family system unit, and changes in the family system unit cause changes in the external environment system. By means of these circular changes and reciprocal relations, the process of family growth and development [7] is created. The chrono system refers to "the time framework with a vector oriented from the present to the future."

The CSFET is characterized by structural distance and functional distance on its horizontal axes and temporal distance on its vertical axis as assessment axes. The structural distance indicates the level of physical/objective separation, and the functional distance, the level of psychological/subjective separation. Through these two dis- 
tances, the relationship between systems can be assessed. The temporal distance refers to the amount of time between separate events, enabling assessment of growth and development of the respective systems.

Nevertheless in Version 2.4 of the CSFET, the position of the nursing professional who provides family care/caring is not clearly established in the family environment. Theoretically the nursing professional would be positioned in the family external environment system, with transactions occurring between the family and nursing professional, and it would be assumed that the relationship between the family and nursing professional could be assessed through the structural distance, functional distance and temporal distance. However, this has not been empirically substantiated. As the CSFET was developed and tested primarily utilizing family ethnography [8], utilizing this method to substantiate family care/caring and implementing it into the family environment of the CSFET will make it possible to update the version of the CSFET.

The aims of this study were 1 ) to empirically substantiate transactions between the family and nursing professional in order to construct a concept of family care/caring, and develop a Family Care/Caring Theory (FCCT); and 2) to improve the CSFET through qualitative and inductive implementation of family care/caring in the CSFET.

\section{Methods}

\subsection{Hypothesizing the Construction of Family Care/Caring Theory}

The basic hypotheses for development of CSFET/FCCT were as follows: The nursing professional is positioned within the family external environment system of the CSFET, and transacts with the family system unit, thereby influencing the family system unit. Conversely, it is provisionally postulated that the family system unit, influenced by the family external environment system, maintains family well-being through its transactions with the nursing professional. The above was structured, a model diagram drawn up, and the CSFET/FCCT draft produced.

\subsection{Family Ethnography}

In order to empirically substantiate the draft of the CSFET/FCCT and ensure its rigor, family ethnography [8] that focused on transactions between the family system unit and the nursing professional was implemented. According to growth and development segments for the family system unit [9], as it is possible for the structure and contents of the CSFET/FCCT to differ, in this study, it was directed at families with a variety of growth and development segments. Further, as the CSFET takes a holistic approach to the family and family environment, research proceeded with guidelines to study families having a variety of sociocultural backgrounds.

\subsubsection{Field Work and Informal Interviews}

In accordance with family ethnography, transactions between the family and nursing professional and notation from the perspective of mutual care were subjected to analysis. Field work was conducted in major urban areas and regional areas of Japan and in Hong Kong, with the informants including a variety of families and nursing professionals.

In the data-collection phase, participant observation, informal interviews and collection of existing materials were conducted. The participant observation was performed at visiting nurse stations, hospitals, private homes and so on. The subjects of informal interviews were individuals at the locations of the participant observations (nurses, public health nurses, midwives, physicians, etc.). Participant observations focused on transactions between families and nursing professionals, and notes were taken of observing and listening to family life and activities, impressions and thoughts by the observers and others. Visual data were collected through shooting of video movies, still photographs and sketches. These were all assembled and utilized to produce field notes.

The data obtained from participant observation and existing materials were confirmed in interviews, and while data obtained in the interviews were confirmed in participant observation and existing materials, multifaceted data collection was conducted, thereby increasing the rigor of the data. As for existing materials, necessary items were extracted from dissertations, books, statistical materials and various recordings.

Field work was conducted in regional areas in Japan by three people for 20 days in 2012 and three people for 16 days in 2013. In major urban areas in Japan, the field work was conducted by three people for 14 days in 2014. In Hong Kong, it was conducted by three people for 11 days in 2012 and by two people for 18 days in 2014. 


\subsubsection{Formal Interviews}

In regional areas in Japan, in 2012 cooperation was obtained from social welfare corporations and high schools. The research plan was distributed and cooperation was requested for the research. Agreement to participate was obtained from 19 families. Then in 2013 cooperation was obtained from general hospitals, clinics, visiting nurse stations and a social welfare council, the research plan was distributed and cooperation requested. Twenty families agreed to participate.

In major urban areas of Japan, in 2014, cooperation was obtained from the community association in a public housing area whose residents were mainly elderly persons, the research plan was distributed and cooperation was requested. Fourteen families agreed to participate.

In Hong Kong, in 2012, cooperation was requested from a physical examination service organization, the middle school of the local Japanese school and a beauty salon operated by a Japanese man. The research plan was distributed to Japanese families residing in Hong Kong and cooperation was requested. Eight Japanese families residing in Hong Kong agreed to participate. Then in 2014 cooperation was once again requested from a physical examination service organization, the research plan was distributed to Japanese families residing in Hong Kong and cooperation was requested. Agreement to participate was obtained from seven families.

Utilizing the draft of the CSFET/FCCT, formal interviews were conducted with the families. The interview guide was focused on transactions between the family and nursing professional, with references to the Family Environment Assessment Index (FEAI) [10] and the Family Environment Map (FEM) [11], tools to assess the transactions between the family environment and family system unit based on the CSFET. A two-hour semistructured interview was conducted with each participant family, which was recorded with the family's permission, and afterwards it was transcribed and made into part of the ethnography data.

At the end of each interview, with references to Graneheim's model of content analysis [12], the transcription was interpreted and analyzed from the perspective of the relationship between the family and nursing professional. Then after making revisions to the CSFET/FCCT the next interview was conducted. To ensure rigor, any questions were clarified and discussed until agreement was obtained among six nursing professionals. Also, in order to clarify the concept of caring, comparisons were made with previously conducted research [13]-[16], and arbitrariness was eliminated with the aim of consistency.

\subsubsection{Construction of CSFET/FCCT}

Based on the ethnographic data obtained as per the above, from categorizing the transactions between the family and nursing professional, the CSFET/FCCT was qualitatively and inductively reconstructed. To ensure rigor, data analysis was repeatedly discussed by two researchers and six practitioners with expert knowledge of family nursing, and their consensus obtained. Through the above process, the development of FCCT and an updated version of the CSFET were completed.

\subsection{Ethical Considerations}

In this study, field work was conducted at each location after gaining approval of the university's Institutional Review Board. When the field work was conducted, the responsible person at the site was given verbal explanation of the purpose of the research and confidential treatment, and permission was obtained for participant observation. The participants at the formal interviews received verbal and written explanations of the purpose of the study, confidential treatment and so on, and agreement to participate was given in writing.

\section{Results}

\subsection{Concept Development of Family Care/Caring and the Construction of Family Care/Caring Theory}

It has been empirically substantiated that from participant observations in the field and formal interviews, a mutually caring relationship exists between the nursing professional and the family system unit. For example, in one case where a family continued caring for a family member at home, until the very end, and a visiting nurse called on the dying person, it can be gleaned from remarks by members of target families such as "She entered into the feelings of family sufferings"; "She was there for us"; and "She watched over us," that the nursing professional had engaged in the practice of family caring while maintaining the appropriate structural distance and 
functional distance. Conversely from the practice of that family caring, it is also clear that the nursing professional is the recipient of caring by the target family. In other words, in this study, the transaction of caring between the family and nursing professional has been empirically substantiated. While it can be supposed that these factors are incorporated into the concept of caring as it currently exists, this study has constructed a new concept of "family care/caring" in transactions between the family and the nursing professional, which had not been clearly demonstrated up to now. Through this study, the concept of family care/caring was constructed and moreover deployed toward Family Care/Caring Theory. That theory is explained below (Figure 1).
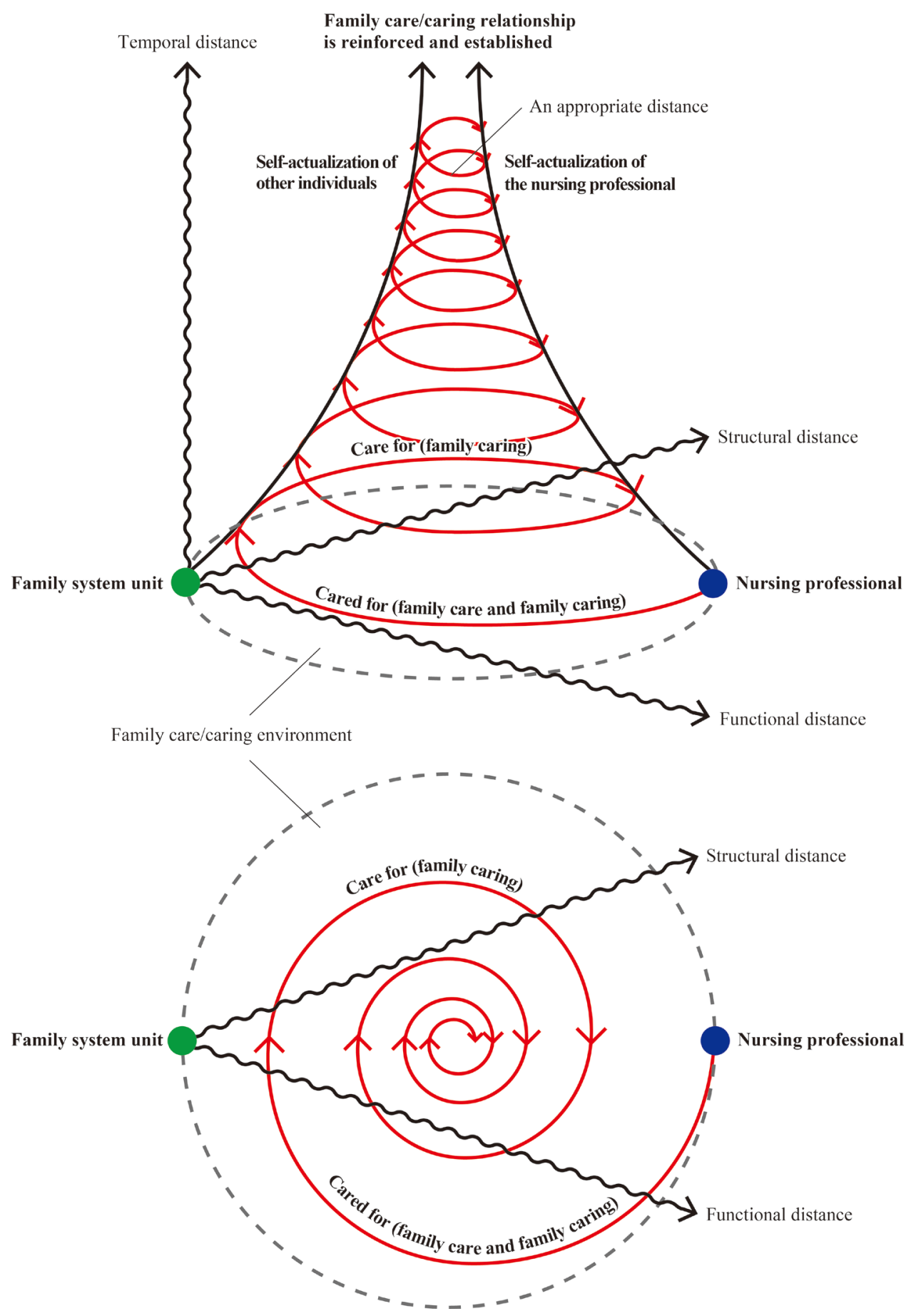

Figure 1. Model diagram of the Family Care/Caring Theory (FCCT) (Ver. 1.0). 
Family care/caring forms the basic principle of family nursing, and is essential for the family system unit to maintain and improve family functioning independently and autonomously. In the family care/caring environment, which is within the family environment, the family system unit is cared for by the nursing professional, and conversely the family system unit cares for the nursing professional. The respective actions are deployed in a circular transaction (mutual act). Looking at the two-dimensional plane formed by the structural distance and functional distance, family caring forms a concentric circular structure and through these transactions, the structural distance and functional distance between the nursing professional and family system unit gradually move closer, maintaining an appropriate distance through deepening of mutual trust. Moreover observing the threedimensional space-time continuum which is created through addition of the temporal distance, family caring forms a helical structure. As transactions are repeated along the temporal axis, the family system unit's self-actualization of other individuals, and the self-actualization of the nursing professional, are realized. Through these processes, a family care/caring relationship is reinforced and established.

In FCCT, family care, a compound noun, is defined as "acts of intervention aimed at maintaining and improving family functioning." Within the phenomena viewed as family care, family caring, a gerund, is defined as "knowing a family's beliefs, intentions and aspirations, to create an attitude of care for that family." Here, beliefs refer to "the way they conduct matters"; intentions refer to "thoughts concerning how they desire matters to be"; and aspirations refer to "things they wish to do." Family caring is guided by a manner of thought that is based on the family system unit in its entirety, and it is necessary for family intervention to focus on non-invasive methods of treatment.

\subsection{Updating the Version of the Concentric Sphere Family Environment Theory}

The CSFET implements the FCCT, and has been updated to Version 2.5 (Figure 2). In other words, "family health care nurses and their colleagues" have been added as an item in the family external environment system, and placed in the macro system of the CSFET. The nursing professional, while engaging in acts with the awareness that he or she is positioned in the family environment, is able to effect transformation of the family and family environment.

The relationship of trust between the family and nursing professional can be clarified by means of the structural distance, functional distance and temporal distance of the CSFET. At that time and place, how the relationship between family and nursing professional is formed, and moreover, the concept of time, that is, how the relationship between family and nursing professional is formed over the passage of time, in other words, it is necessary to give attention to the temporal distance of the CSFET. In the practice of family caring, an understanding of the FCCT and CSFET will be necessary.

\section{Discussion}

In this study, first, a conceptual meaning for family care/caring was created. The creation of a conceptual meaning is an approach to theory-building [17]. In other words, it undergoes a process of construction of FCCT from the creation of conceptual meaning. FCCT is a middle-range family nursing theory to explain a nursing phenomenon in which a relationship is created between the family receiving family care and the nursing professional who provides such care. This focuses on the process of establishing a family care/caring relationship between the family and the nursing professional. The mapping out of this theory is the Family Care/Caring Model (FCCM) as shown by the three-dimensional structural diagram (Figure 1) provided to facilitate visual comprehension. The degree of perception of distance in interpersonal relationships is represented by the concept of functional distance, but the relationship between the family system unit and family environment is not only the functional distance; it is also necessary to consider the structural distance, which refers to the degree of physical separation [7]. In the FCCT, which is based on the CSFET, the relationship of trust between the family and nursing professional can be clarified by the structural distance and functional distance. Through future use in clinical settings its effectiveness will be empirically substantiated, and it will be necessary to continuously engage in creative revisions.

The FCCT is constructed by layers of research by means of family ethnography. The family environment was reviewed over a wide scope, and it went through the process of development based on a diverse data that were obtained through permeation of family life and ways of thinking. Consequently it is believed that the objective, of a holistic approach to transactions between the family and nursing professional, was achieved. 


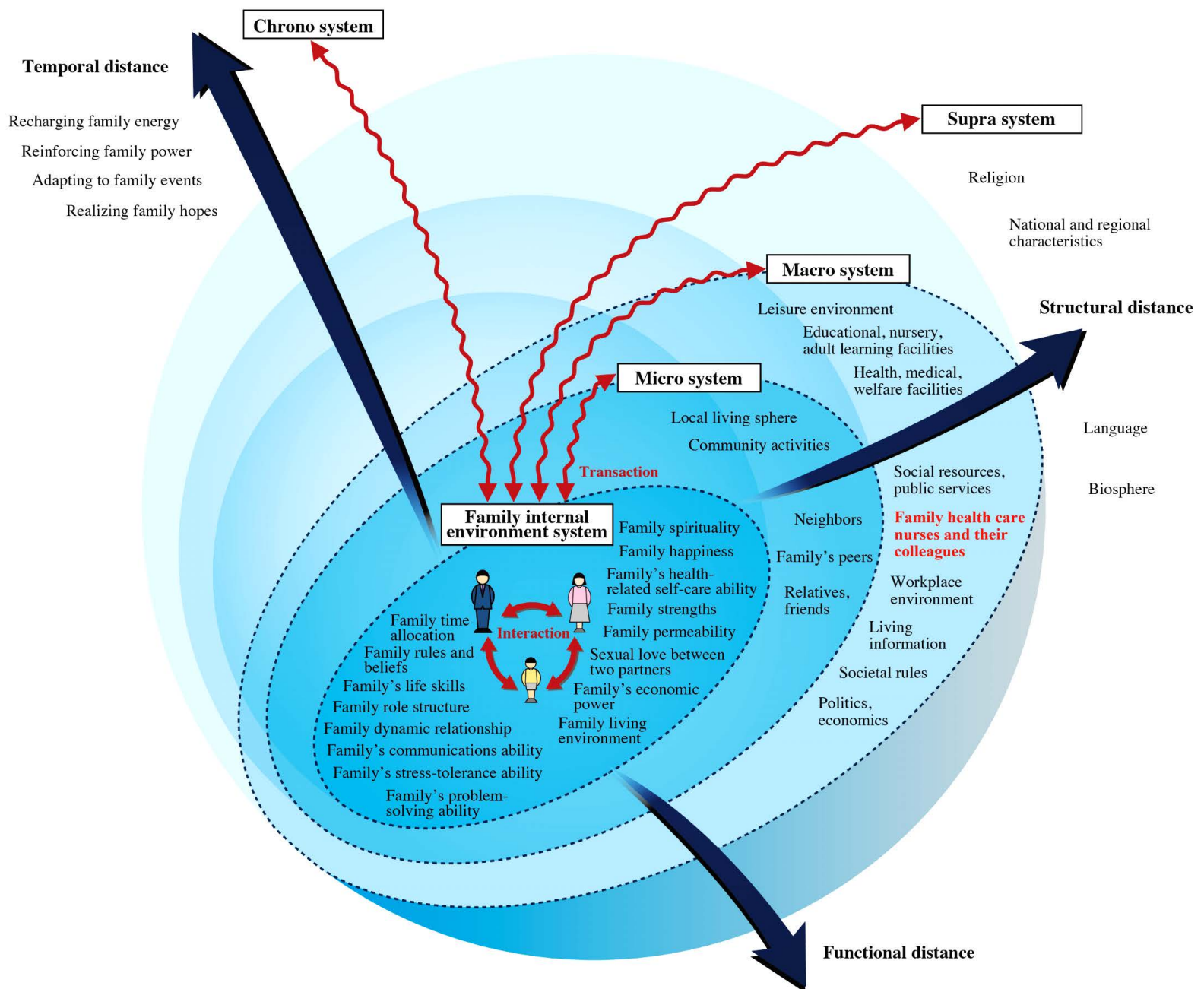

Figure 2. Model diagram of Concentric Sphere Family Environment Theory (CSFET) (Ver. 2.5).

Family nursing carries the connotation of family care and family caring, and it is believed that the quality of family nursing is determined by what sort of relationship built between the nursing professional and the family. In the CSFET/FCCT, through readiness to become close to the family, in other words, through empathy in understanding of the family and its world, it is important that a situation be constructed of "being together with the family." Moreover, the nursing professional, by self-knowledge, can achieve self-elevation, which is where the practice of family caring comes to be.

From the standpoint of the nursing professional, family caring promotes the growth and development of the family system unit, and improves family functioning (i.e., the self-actualization of other individuals). The nursing professional, on the other hand, realizes self-healing and self-fulfillment is promoted (i.e., the self-actualization). In this manner, the phenomena that develop between the family and nursing professional are the phenomena of family caring. The outcome of family care/caring is 1) self-actualization of other individuals; 2) selfactualization; and 3) establishment of a family caring relationship. In order to make for an effective and high quality moments of family intervention, which never recur twice in the same setting, establishment of a family caring relationship is a prerequisite.

The nursing professional feels "concern" toward the target family in his or her care, and must possess a clear desire for the family to realize its hopes to the greatest degree possible. The family care/caring environment is the family environment, the shared venue in which the family and nursing professional engage in family care/ caring. It is necessary to also decide the direction of the functional distance between the family and nursing professional. In other words, in terms of functional distance as seen by the nursing professional, there is the func- 
tional distance by which the nursing professional cares for the family, and the functional distance by which the nursing professional is cared for by the family. The former is defined as the "active functional distance," while the latter is defined as the "passive functional distance," and the two are not necessarily of the same length. For example, in the case of family intervention that does not affect family beliefs [18], intentions and aspirations, active functional distance becomes overly shorter irrespective of the passive functional distance. In this case, it is necessary for the nursing professional to endeavor to build a relationship of trust.

\section{Conclusion}

The Family Care/Caring Theory (FCCT) was developed for the purpose of caring for the family system unit. This was implemented into the Concentric Sphere Family Environment Theory (CSFET), an existing family nursing theory. In the family care/caring environment, the "act" by which the nursing professional engages in family intervention on a concerned family with which he or she is dealing is family care. Then in the context of the culture of the target family, family care is provided, and the "attitude" by which intervention can enable a family to live on its own terms is family caring. For the practice of family caring, an understanding of FCCT and the CSFET will be indispensable.

\section{Acknowledgements}

The authors sincerely appreciate analytical assistance provided by Ms. Saina Taniguchi, Ms. Saori Komiya, Ms. Ai Washizu, and Mr. Hirofumi Mizukami, Kobe University. This project was supported in part by a Grant-inAid for Scientific Research (B) from the Japan Society for the Promotion of Science (JSPS) in Japan (Grant Nos. 24390498 and 15H05084).

\section{Declaration of Conflicting Interests}

The authors declare that they have no competing interests.

\section{References}

[1] Noddings, N. (2013) Caring: A Relational Approach to Ethics and Moral Education. 2nd Edition, University of California Press, Berkeley.

[2] Hohashi, N. and Honda, J. (2010) Family Functioning. In: Hohashi, N., Ed., New Family Health Care Nursing: Theory, Practice and Research, Medical Friend Sha, Tokyo, 38-45.

[3] Honda, J. and Hohashi, N. (2015) The Trend of Research Concerning “Caring” in Japan. International Journal for Human Caring, 19, In Press.

[4] Ray, M.A. (1989) The Theory of Bureaucratic Caring for Nursing Practice in the Organizational Culture. Nursing Administration Quarterly, 13, 31-42. http://dx.doi.org/10.1097/00006216-198901320-00007

[5] Hohashi, N. and Honda, J. (2014) “Unreciprocated Family Members” Issue (Hohashi). In: Hohashi, N., Ed., Assessment Guide for Japanese Version of the Family Environment Map (FEM-J), Editex, Tokyo, 27-29.

[6] Hohashi, N. and Kobayashi, K. (2010) Methods of Treatment of the Family System Unit. In: Hohashi, N., Ed., New Family Health Care Nursing: Theory, Practice and Research, Medical Friend Sha, Tokyo, 16-25.

[7] Hohashi, N. and Honda, J. (2011) Development of the Concentric Sphere Family Environment Model and Companion Tools for Culturally Congruent Family Assessment. Journal of Transcultural Nursing, 22, 350-361. http://dx.doi.org/10.1177/1043659611414200

[8] Honda, J. and Hohashi, N. (2010) Ethnography. In: Hohashi, N., Ed., New Family Health Care Nursing: Theory, Practice and Research, Medical Friend Sha, Tokyo, 399-404.

[9] Honda, J. and Hohashi, N. (2014) Development of a New Growth and Development Sectors for the Family System Unit. Proceedings of the Sigma Theta Tau International's 25th International Nursing Research Congress, Hong Kong, 25 July 2014, 822-823.

[10] Hohashi, N. and Honda, J. (2013) Japanese Version of the Family Environment Assessment Index (FEAI-J). Editex, Tokyo.

[11] Hohashi, N. and Honda, J. (2015) Assessment Guide for the English Version of the Family Environment Map (FEM-E). Editex, Tokyo.

[12] Graneheim, U.H. and Lundman, B. (2004) Qualitative Content Analysis in Nursing Research: Concepts, Procedures 
and Measures to Achieve Trustworthiness. Nurse Education Today, 24, 105-112. http://dx.doi.org/10.1016/j.nedt.2003.10.001

[13] Purnell, M.J. (2006) Development of a Model of Nursing Education Grounded in Caring and Application to Online Nursing Education. International Journal for Human Caring, 10, 8-16.

[14] Emoto, R., Tsutsui, M. and Kawana, R. (2015) A Model to Create a Caring and Healing Environment for Nurses in Child and Family Nursing. International Journal for Human Caring, 19, 8-12.

[15] Ray, M.A. (2009) Transcultural Caring Dynamics in Nursing and Health Care: The Dynamics of Contemporary Nursing. F.A. Davis Company, Philadelphia.

[16] Smith, M.C., Turkel, M.C. and Wolf, Z.R. (Eds.) (2012) Caring in Nursing Classics: An Essential Resource, Springer Publishing Company, New York.

[17] Chinn, P.L. and Kramer, M.K. (2011) Integrated Theory and Knowledge Development in Nursing. 8th Edition, Mosby, St. Louis.

[18] Wright, L.M. and Bell, J.M. (2009) Beliefs and Illness: A Model for Healing. 4th Floor Press, Calgary. 